\title{
Stimuli-Sensitive Poly(NIPA-co-APA) Hydrogels for the Controlled Release of Keterolac Tromethamine
}

\author{
Yonghyun Kim, V. Ramesh Babu, K. S. V. Krishna Rao ${ }^{\dagger}$ Jae-Min Lim, \\ T. Daniel Thangadurai ${ }^{\ddagger}$, and Yong-IIl Lee* \\ Anastro Laboratory, Department of Chemistry, Changwon National University, Changwon 641-773, Korea. \\ *E-mail: yilee@changwon.ac.kr \\ ${ }^{\dagger}$ Department of Chemistry, Yogi Vemana University, Kadapa-516003, India \\ ${ }^{\dagger}$ Department of Nanotechnology, College of Engineering, Sri Ramakrishna Institutions, Coimbatore 641022, India
}

(Received November 25, 2013; Accepted January 3, 2014)

\begin{abstract}
ABSTRCT. The pH sensitive hydrogels composed of $N$-isopropylacrylamide (NIPA) and acryloyl phenylalanine (APA) were prepared by redox polymerization using $N, N^{\prime}$-methylenebisacrylamide (MBA) as a crosslinker. Anti-inflammatory and analgesic agent, Keterolac Tromethamine (KT), was loaded successfully into poly(NIPA-co-APA) copolymeric hydrogels by swelling equilibrium method. To understand the nature of drug in the polymeric matrix, the newly synthesized drug loaded poly(NIPAco-APA) copolymeric hydrogels were characterized by using differential scanning calorimetry (DSC) and X-ray diffraction (XRD) techniques. The scanning electron microscopy (SEM) technique result indicates the spherical smooth surface of the hydrogels. The drug (KT) releasing nature of the poly(NIPA-co-APA) hydrogels was studied in pH 1.2 and 7.4. Effects of drug loading, crosslinking agent, $\mathrm{pH}$ and the ionic strength of the external medium on swelling of hydrogels were also investigated.
\end{abstract}

Key words: Hydrogel, Keterolac tromethamine, Controlled Release, Stimuli-responsive

\section{INTRODUCTION}

In recent years, the incorporation of amino acids into synthetic polymers has been acknowledged a considerable interest because it can lead to new biomaterials with a wide range of properties that can be easily modulated by varying the components in the building block of the macromolecular backbone during synthesis. ${ }^{1-5}$ Examples of amino acid-derived organic polymers based on polyacrylates, polyisobutylenes, polyurethanes, polyacetylenes, and hydrogels have been developed for diverse applications including controlled drug delivery systems, antibacterical activity, affinity based separators, and optobioelectronic devices. ${ }^{6-12}$

Hydrogels have been an extensive research topic during last several decades due to their high water content and the possible control over the swelling kinetics make them very attractive for biomedical applications. Owing to their widely variable and adjustable properties, hydrogels based on synthetic polymers have principally been studied as the vehicles for the controlled release of both low-molecular mass drugs and macromolecular drugs including therapeutic proteins, enzymes, and DNA. ${ }^{13}$ The gel properties like porosity, swelling behavior, stability, biodegradability, gel strength, and biocompatibility may be tailored for a particular application. A special class of hydrogels, which are called "intelligent" or "stimuli-responsive" hydrogels, exhibits significant volume change in response to small changes in external stimuli, such as $\mathrm{pH}$, temperature, light, etc. The most interesting $\mathrm{pH}$ - and temperature-sensitive "intelligent" hydrogels have extensively been used in the development of drug delivery systems for pharmaceutical applications.

Poly $\left(N^{\prime}\right.$-isopropylacrylamide) (PNIPA) hydrogel which is an intelligent hydrogel with a lower critical solution temperature (LCST) $)^{14-18}$ can be used for drug delivery systems, bioseparation, and other biotechnology applications..$^{19,20}$ The volume phase transition of PNIPA matrix at $32^{\circ} \mathrm{C}$ is narrow and not practical at physiological temperature $\left(37^{\circ} \mathrm{C}\right)$. It is easy to tune the release of drug by temperature- and $\mathrm{pH}$-sensitive systems due to the wide range of physiological $\mathrm{pH}$ mean values (2.20 in stomach to 6.80 in intestine) ${ }^{21}$ The thermosensitivity of hydrogel could be controlled with $\mathrm{pH}$ by incorporating the small amount of ionizable groups (such as carboxyl) into PNIPA chains. ${ }^{22}$

Ketorolac tromethamine (KT, Scheme 1) is a non-steroidal anti-inflammatory drug with 4-6 h of biological halflife period. The recommended total daily dose of KT tablets (maximum $40 \mathrm{mg}$ ) is significantly lower than KT injection (maximum $120 \mathrm{mg}$ ). Dosage should be adjusted for patients 65 years or older, for patients under $50 \mathrm{~kg}(110 \mathrm{lbs})$ of body weight and for patients with moderately elevated 
<smiles>O=C(c1ccccc1)c1ccc2n1CCC2C(=O)O</smiles>

Scheme 1. Chemical structure of Ketorolac Tromethamine (KT).

serum creatinine. Furthermore, doses of KT injection are not to exceed $60 \mathrm{mg}$ (total dose per day) in these patients. Therefore, it is necessary to enhance sustain action of drug for longer periods. If KT administrates directly as a conventional formulation, it causes gastro intestinal complications including irritation, ulcer, bleeding, and perforation. ${ }^{23,24}$ Therefore, it is essential to develop a $\mathrm{pH}$ sensitive controlled drug delivery system to prevent the above problems. The present communication reports the development of amino acid based $\mathrm{pH}$-sensitive hydrogels for the controlled release of drug KT. Swelling studies as well as drug release studies have been performed at $\mathrm{pH} 1.2$ and 7.4 to meet gastro intestinal conditions.

\section{EXPERIMENTAL}

\section{Materials}

Ketorolac tromethamine (KT), $N$-isopropylacrylamide (NIPA), $N, N^{\prime}$-methylenebisacrylamide (MBA), ammonium persulfate (APS) were purchased from Aldrich. $N, N, N^{\prime}, N^{\prime}$ tetramethylethylenediamine (TEMED) was purchased from Alfa Aesar A Johnson Matthey Company. $N$-acryloyl-Lphenylalanine (APA) was synthesized by the procedure given in prior art. $^{25}$

\section{Preparation of Dissolution Media}

The buffer solution at $\mathrm{pH} 1.2$ was prepared by taking $50 \mathrm{~mL}$ of $0.2 \mathrm{M} \mathrm{KCl}$ and $7.8 \mathrm{~mL}$ of $0.2 \mathrm{~N} \mathrm{HCl}$ in volumetric flask and diluted to $200 \mathrm{~mL}$ with distilled water. The $\mathrm{pH} 7.4$ solution was prepared by taking 50 $\mathrm{mL}$ of $0.2 \mathrm{M} \mathrm{KH}_{2} \mathrm{PO}_{4}$ and $39.1 \mathrm{~mL}$ of $0.2 \mathrm{~N} \mathrm{NaOH}$ in volumetric flask and diluted to $200 \mathrm{~mL}$ with distilled water. $^{26}$

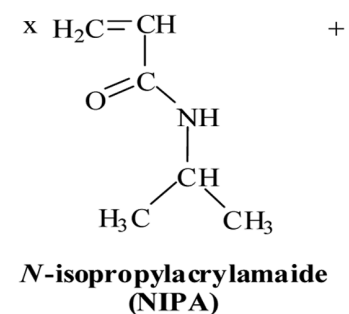
(NIPA)

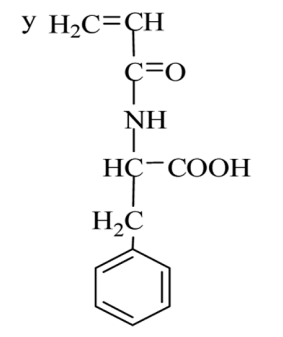

Acryloyl phenyl alanine (APA)<smiles>C=CC(=O)NCNC(=O)C=C</smiles>

$N, N^{\prime}$-methylenebisacrylamide (MBA)

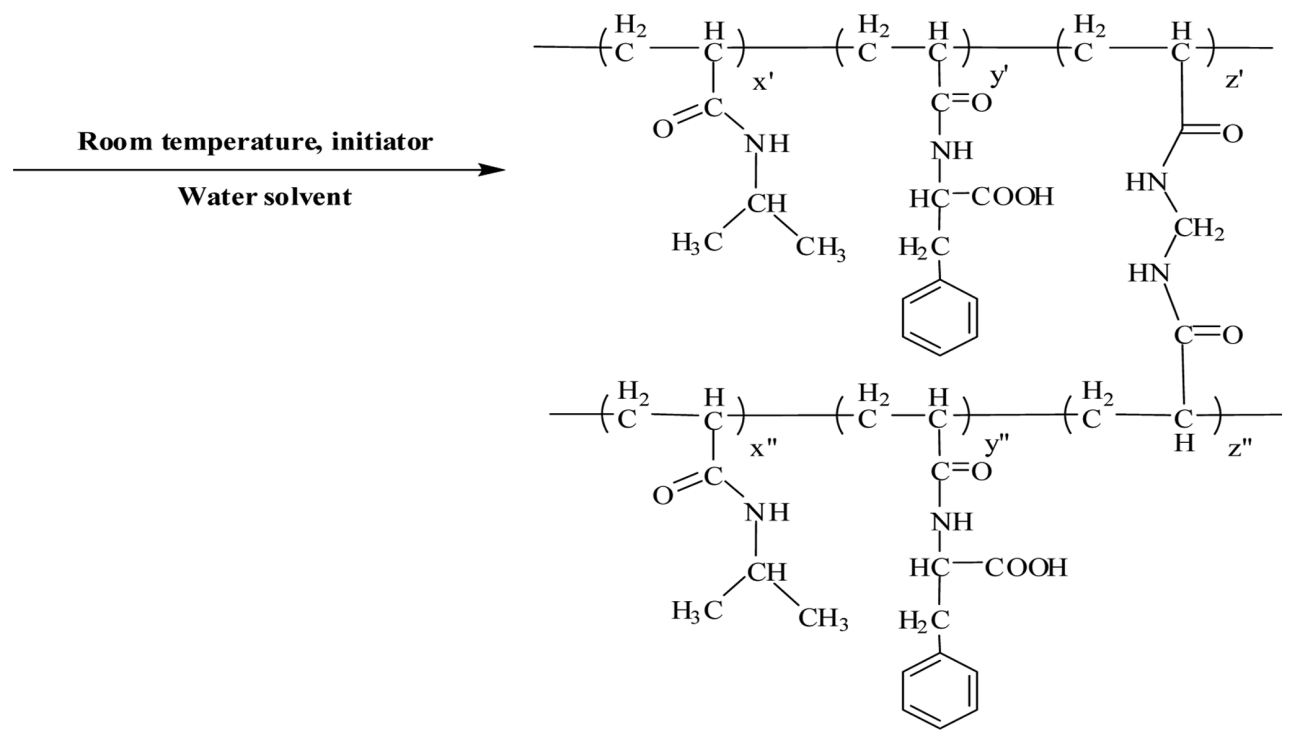

Polv(NIPA-co-APA) hvdrogel

Scheme 2. Synthetic scheme of poly(NIPA-co-APA) hydrogel. 
Synthesis of Poly( $N$-isopropyl acrylamide-co-acryloyl
phenyl alanine), Poly(NIPA-co-APA), Hydrogels

Poly(NIPA-co-APA) hydrogels were prepared by redox polymerization. NIPA ( $800 \mathrm{mg}$ ), APA (200 mg), and MBA $(10 \mathrm{mg})$ were dissolved in $4 \mathrm{~mL}$ of distilled water at room temperature (APA dissolved in equimolar ratio of $\mathrm{NaOH}$ ). To this homogeneous monomer solution, initiator APS (10 $\mathrm{mg})$ and accelerator TEMED $(50 \mu \mathrm{L})$ were added sequentially under continuous stirring. The free-radical crosslinking polymerization was continued for $24 \mathrm{~h}$ at $5{ }^{\circ} \mathrm{C}$ in order to complete the network formation. The reaction mechanism for the synthesis of hydrogels has been illustrated in Scheme 2 . The synthesized copolymeric hydrogels were taken out and immersed in distilled water for 3 days at room temperature by changing the water every $12 \mathrm{~h}$ in order to remove residual unreacted monomers. The resulting "swollen" hydrogels were dried in air for 2 days and then in a vacuum oven at $37^{\circ} \mathrm{C}$ until attaining a constant weight. The copolymeric hydrogels were prepared by varying the ratios of monomers, the amount of crosslinking agent, and the different amount of drug. The pure PNIPA hydrogel without APA was also fabricated under the same condition. All formulations used in this work were given in Table 1.

\section{Swelling Studies}

Dynamic swelling of the copolymeric hydrogels prepared with three different crosslink densities as well as three different drug loadings was studied at $\mathrm{pH} 1.2$ and 7.4 by mass measurements at $37^{\circ} \mathrm{C}$. To perform the swelling experiments, hydrogels were soaked in water and then removed from the swelling bottles at different time intervals. The hydrogels were wiped carefully with tissue paper (without pressing hard) to remove the surface-adhered water. Swelling experiments were repeated thrice for each sample and the average values were used in data analysis. The standard deviations (SD) in all cases were $<5 \%$. The percentage of swelling ratio ( $\% \mathrm{SR})$ was calculated by the following equation (1).

$$
\% S R=\left(\frac{W_{s} \times W_{d}}{W_{d}}\right) \times 100
$$

where, $W_{s}$ is the weight of the swollen gel at time $t$ and $W_{d}$ is the dry weight of the gel.

\section{Release Dynamics of the Model Drug Standard curve of Ketorolac Tromethamine}

In this procedure, the absorbance of a number of standard solutions with the reference substance encompassing the sample concentrations were measured by using the UV-vis Spectrophotometer (Agilent 8453 UV spectrophotometer) at $\lambda_{\max } 324 \mathrm{~nm}$. The calibration graph was constructed and the concentration of the drug in sample solution was determined from the absorbance of solution. Two calibration graphs were made in $\mathrm{pH} 1.2$ and 7.4 dissolution media, to determine the amount of drug release from the drug-loaded hydrogels.

\section{Loading of the drug in copolymeric networks}

The loading of the drug onto poly(NIPA-co-APA) hydrogels was carried out by the swelling equilibrium method. The poly(NIPA-co-APA) hydrogels were allowed to swell in the drug solution of known concentration for $24 \mathrm{~h}$ at $37^{\circ} \mathrm{C}$ and then dried to obtain the release device. ${ }^{27}$

\section{Estimation of drug loading and encapsulation}

The loading efficiency of KT in the copolymeric hydrogel was examined spectrophotometrically. About $10 \mathrm{mg}$ of the drug-loaded hydrogels were placed in $10 \mathrm{~mL}$ of buffer solution and stirred vigorously for $48 \mathrm{~h}$ to extract drug from the hydrogels. The solution was filtered and assayed by using the UV spectrophotometer at $324 \mathrm{~nm}$. The percentage (\%) drug loading and encapsulation efficiency were calculated by Eqs. (2) and (3), respectively, and the data were compiled in Table 1.

Table 1. Formulation of hydrogels with $\mathrm{KT}$ and their encapsulation efficiencies

\begin{tabular}{cccccccc}
\hline Sample code & NIPA $(\mathrm{g})$ & APA $(\mathrm{g})$ & MBA $(\mathrm{wt} \%)$ & Drug $(\mathrm{mg})$ & APS $(w t \%)$ & TEMED $(\mu \mathrm{L})$ & \% Encapsulation Efficiency \\
\hline NAD 0 & 0.8 & 0.2 & 1 & 0 & 1 & 50 & 0 \\
NAD 1 & 0.7 & 0.3 & 1 & 50 & 1 & 50 & 62.3 \\
NAD 2 & 0.8 & 0.2 & 1 & 50 & 1 & 50 & 55.2 \\
NAD 3 & 0.9 & 0.1 & 1 & 50 & 1 & 50 & 50.5 \\
NAD 4 & 0.8 & 0.2 & 2 & 50 & 1 & 50 & 46.7 \\
NAD 5 & 0.8 & 0.2 & 3 & 50 & 1 & 50 & 61.1 \\
NAD 6 & 0.8 & 0.2 & 1 & 100 & 1 & 50 & 73.7 \\
NAD 7 & 0.8 & 0.2 & 1 & 150 & 1 & 50 & 46.5 \\
NAD 8 & 1 & 0 & 1 & 50 & 1 & 50 & \\
\hline
\end{tabular}


$\%$ Drug loading $=$

$\left(\frac{\text { Amount of drug in the P(NIPA-co-APA) hydrogels }}{\text { Amount of P(NIPA-co-APA) hydrogels }}\right) \times 100$

$\%$ Encapsulation efficiency $=\frac{\text { Actual loading }}{\text { Theoretical loading }} \times 100$

\section{In vitro release studies}

The KT release from the poly(NIPA-co-APA) copolymeric hydrogels was investigated in dissolution media at $\mathrm{pH} 1.2$ and 7.4 with different percentage of KT loading and different extent of crosslinking. In vitro release experiments were performed by using the digital tablet dissolution test apparatus (Model: VDA-8D, Mumbai, India) at a stirring speed of $100 \mathrm{rpm}$. The weighed quantities of the samples equivalent to $10 \mathrm{mg}$ of the drug were placed in the basket, which was then immersed in $900 \mathrm{~mL}$ dissolution medium maintained at $37^{\circ} \mathrm{C}$. A $5 \mathrm{~mL}$ of sample aliquot was withdrawn at different time intervals and filtered through a $0.25 \mu \mathrm{m}$ filter. The dissolution media were then replaced with $5 \mathrm{~mL}$ of fresh dissolution media. The KT concentration was determined spectrophotometrically at $324 \mathrm{~nm}$. These measurements were carried out in triplicate for each sample, but average values were considered in data analysis and graphical presentations.

\section{Characterization}

\section{Differential scanning calorimetric analysis}

Differential scanning calorimetry (DSC) was performed on placebo poly(NIPA-co-APA) hydrogel, drug-loaded poly(NIPA-co-APA) hydrogel, and pristine KT. The thermal properties of poly(NIPA-co-APA) copolymeric hydrogels were evaluated by using TA 5000/SDT 2960 DSC Q10 thermal system (Zurich, Switzerland). About 1-4 mg of sample was heated from 25 to $250{ }^{\circ} \mathrm{C}$ at the heating rate of $10^{\circ} \mathrm{C} / \mathrm{min}$ under nitrogen atmosphere with keeping the flow rate at $20 \mathrm{~mL} / \mathrm{min}$.

\section{X-ray diffractometry (XRD)}

The crystallinity of KT after encapsulation was evaluated for placebo poly(NIPA-co-APA) hydrogel, drug-loaded poly (NIPA-co-APA) hydrogel, and pristine KT by using the X-ray diffractometer (X'pert MPD 3040). The uniform size of dried poly(NIPA-co-APA) hydrogel was mounted on a sample holder and XRD patterns were recorded in the angle range of $5-45^{\circ}$ at the speed of $5 \% \mathrm{~min}$.

\section{Scanning Electron Microscopy (SEM)}

SEM images of the poly(NIPA-co-APA) hydrogels were recorded using a MIRA LMH, H.S. scanning electron microscope (SEM), equipped with Phoenix energy dispersive analysis of X-rays (EDAX) at the required magnification. The micrographs were obtained at $15 \mathrm{kV}$ as operating voltage.

\section{RESULTS AND DISCUSSION}

\section{Differential Scanning Calorimetric Study}

The DSC thermograms of (a) placebo poly(NIPA-co-APA) hydrogels, (b) drug-loaded poly(NIPA-co-APA) hydrogels, and (c) pristine KT are presented in Fig. 1. The crystallinity of drug and the melting temperature $\left(T_{m}\right)$ of polymer were determined. The placebo poly(NIPA-co-APA) hydrogels have shown an endothermic peak at $96^{\circ} \mathrm{C}$, indicating the melting temperature, whereas the drug-loaded poly(NIPA-co-APA) hydrogels showed an endothermic peak at $85^{\circ} \mathrm{C}$. However, a strong exothermic decomposition peak was observed around $172{ }^{\circ} \mathrm{C}$ for KT, which designates the melting and the decomposition of the drug. It should be noted that this exothermic peak did not appeared in all the drug-loaded hydrogels comfirming an amorphous dispersion of the drug into the polymer matrix.

\section{X-ray Diffraction Studies}

To investigate the crystallinity of the drug in the crosslinked poly(NIPA-co-APA) hydrogels, x-ray diffraction patterns of Placebo poly(NIPA-co-APA) hydrogels, drug-loaded poly(NIPA-co-APA) hydrogels and pristine KT were recorded (Fig. 2a-c). Characteristic intense peaks were observed between $8,14,18$, and $20^{\circ}$ due to crystalline nature of KT (Fig. 2c). However, these peaks were disappeared when KT was loaded in poly(NIPA-co-APA) hydrogels and only

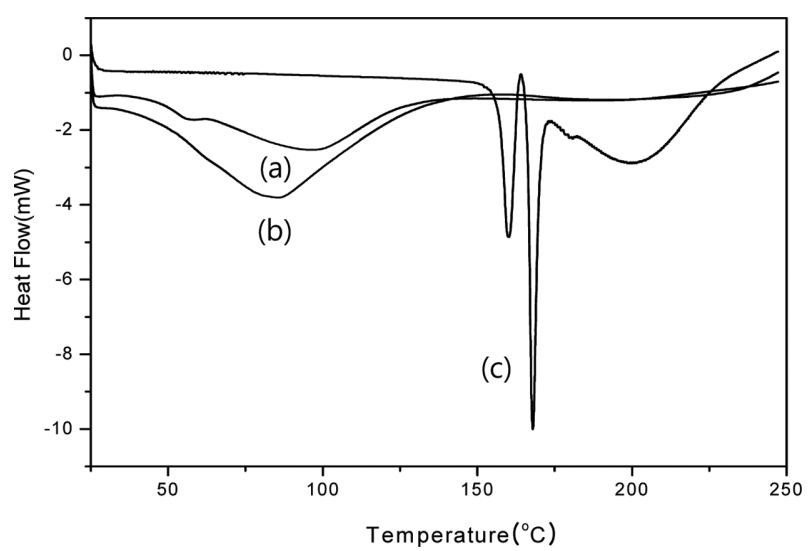

Figure 1. DSC thermograms of (a) placebo poly(NIPA-co-APA) hydrogels, (b) drug-loaded poly(NIPA-co-APA)hydrogels, and (c) pristine KT. 


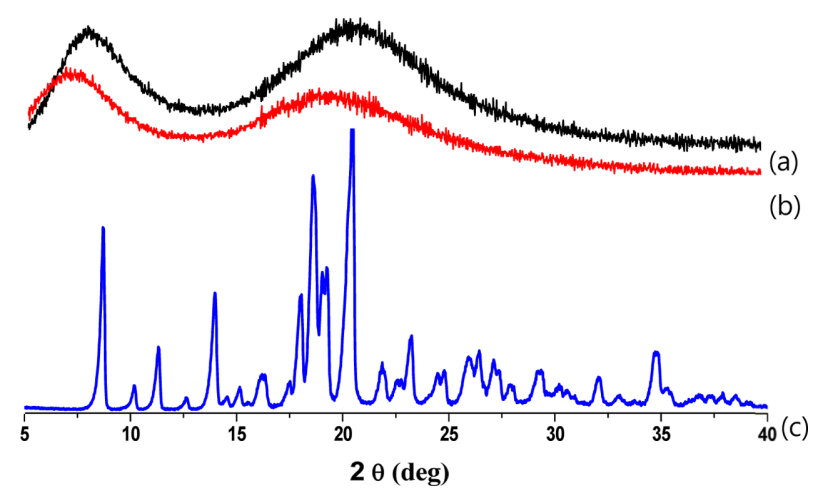

Figure 2. X-ray diffraction patterns of (a) placebo poly(NIPAco-APA) hydrogels, (b) drug-loaded poly(NIPA-co-APA) hydrogels, and (c) pristine KT.

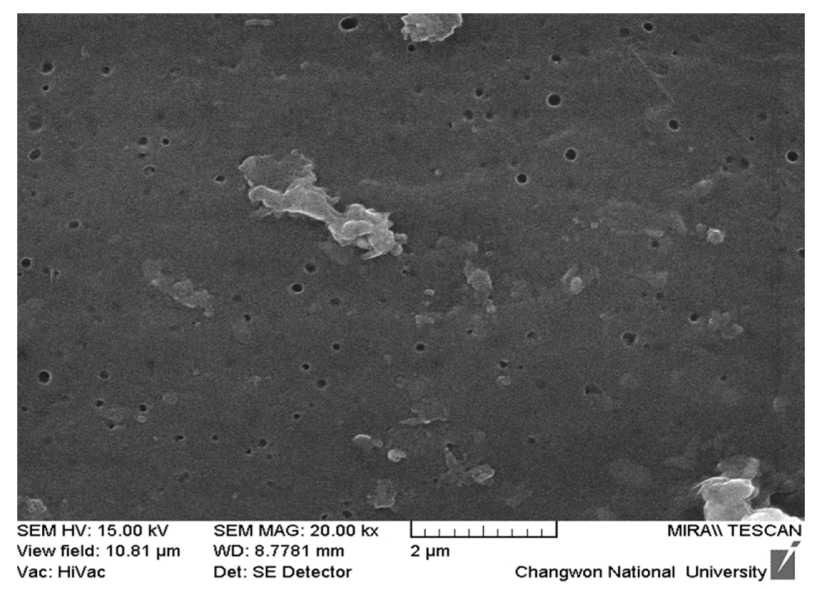

Figure 3. Scanning Electron Microscope image of a pure poly (NIPA-co-APA) hydrogel.

placebo polymer matrix peaks were observed (Fig. 2a,b). This result revealed that the KT dispersed well at the molecular level in the polymer matrix and crystallinity was not found in the drug-loaded matrices. Moreover, it is very difficult to measure the detection limit due to the amorphous nature of the loaded drug.

\section{Scanning Electron Microscopy Studies}

The SEM micrographs of poly(NIPA-co-APA) hydrogels indicate smooth surface with partial holes which helps the drug release as well as swelling capacity of the hydrogels (Fig. 3).

\section{Swelling Studies}

Hydrogels are composed of hydrophilic homopolymer or copolymer networks, which can swell in the presence of water or physiological fluids. Swelling parameters are the most important factors for hydrogel characterization because of fundamental relationship exists between the swelling of a polymer and the nature of the swelling medium. ${ }^{28,29}$ Chemical crosslinks (covalent bonds) or physical junctions (e.g., secondary forces, crystallite formation, chain entanglements) provide the hydrogels unique swelling behavior and three-dimensional structure. ${ }^{30-32}$ In order to evaluate the optimum reaction parameters for the synthesis of hydrogels, we took the swelling of the hydrogels and the structural integrity maintained by the hydrogels after swelling for $24 \mathrm{~h}$ as the criteria. The swelling of the poly(NIPA-co-APA) hydrogels was studied as a function of APA content, in $\mathrm{pH}$ value of 1.2 and 7.4, amount of KT and amount of MBA in the polymer matrix.

\section{Effect of $\boldsymbol{N}$-acryloyl-L-phenylalanine (APA) contents}

The effect of monomer concentration on the swelling of crosslinked polymers was investigated as a function of APA contents and the results are presented in Fig. 4(a). The extent of APA is related largely to the swelling equilibrium. It was observed that the $\%$ swelling ratios of the hydrogels were increased from 44 to 1652 with increasing the amount of APA from 0 to $300 \mathrm{mg}$. This is attributed to increase the hydrophilicity of matrix due to the presence of carboxylic as well as amide groups in APA.

\section{Effect of drug loading}

The hydrogels were prepared with different KT contents from 0 to $150 \mathrm{mg}$ in order to study the entrapment of $\mathrm{KT}$ on the polymer network. The swelling was taken in $\mathrm{pH}$ 7.4 dissolution media at $37^{\circ} \mathrm{C}$. As can be seen in Fig. 4(b), the swelling was increased with increasing KT contents in the composition of hydrogel matrix. Such an increase in swelling of the matrix is mainly due to the incorporation of the acidic nature of KT and formation of matrix between hydrophilic APA and NIPA chains.

\section{Effect of crosslinking agent}

The effect of crosslinking agent (MBA) on the swelling of the hydrogel at $37^{\circ} \mathrm{C}$ was investigated by preparing polymers with different concentration of crosslinking agent (Fig. 4(c)). The extent of crosslinking is dependent upon the amount of crosslinking agent (MBA) used and the swelling equilibrium. For instance, \% swelling ratio decreased from 1415 to 897 with increasing the amount of MBA from 10 to $30 \mathrm{mg}$. The denser crosslinking produced by increasing the crosslinker concentration leading to decreased void size in the polymer matrix and thereafter decreases the swelling in the polymer.

\section{Effect of $\mathbf{p H}$}

To investigate the effect of $\mathrm{pH}$ and ionic strength of the 

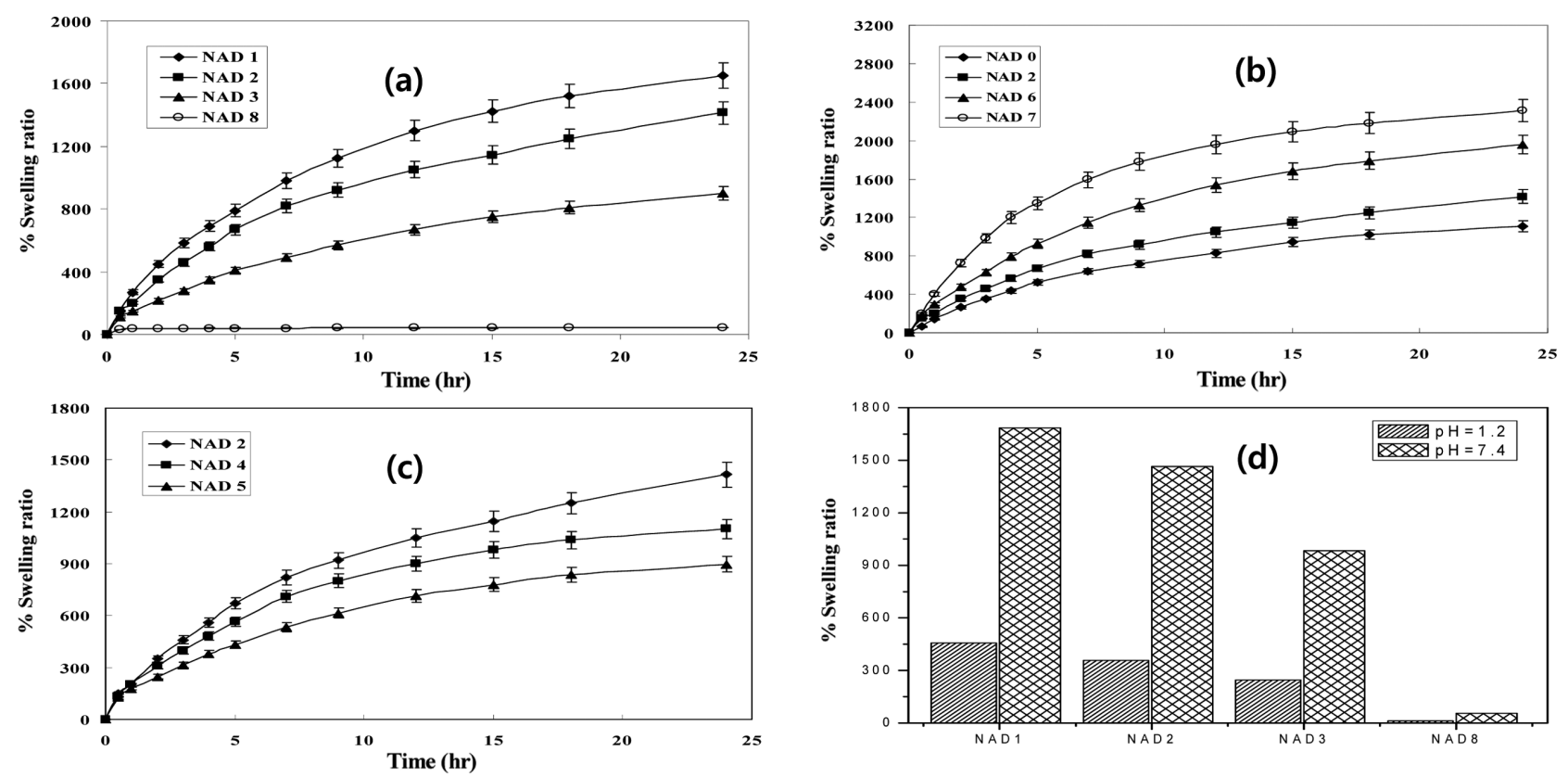

Figure 4. Effect of (a) N-acryloyl-L-phenylalanine (APA), (b) drug loading, (c) crosslinking agent in distilled water, and (d) $\mathrm{pH}$ in different buffer medium, on swelling kinetics of poly(NIPA-co-APA) hydrogels at $37^{\circ} \mathrm{C}$.

external medium on the swelling of poly(NIPA-co-APA) hydrogels, the swelling experiments were carried out in $\mathrm{pH}$ 1.2 and 7.4 (Fig. 4(d)). The \% swelling ratio values were found to be much higher at $\mathrm{pH} 7.4$ than at 1.2 due to the swelling ratio of the hydrogel was limited at $\mathrm{pH} 1.2$ owing to the formation of intermolecular hydrogen bonds. At $\mathrm{pH}$ 7.4, the carboxylic acid groups on the hydrogel become ionized (-COO-) progressively. In this case, a large swelling force created by the electrostatic repulsion between the ionized acid groups make the hydrogel to be swelled more significantly. The electrostatic attraction between opposite charged molecules is an adjustable driving force for structured material construction.

\section{Encapsulation Efficiency (EE \%)}

In order to explore the percentage of encapsulation efficiency (EE \%), three different concentrations of $\mathrm{KT}(1,2$, and $3 \mathrm{wt} \%$ ) were loaded in the hydrogels. The results reveal that the percentage of encapsulation efficiency increases by increasing the drug concentration (Table 1). It should be noticed that $\mathrm{EE} \%$ was enhanced with increasing the amount of APA in the copolymeric hydrogels. The copolymeric hydrogels containing $0,0.1,0.2$, and $0.3 \mathrm{~g}$ of APA, and $1 \mathrm{wt} \%$ of KT with $1 \mathrm{wt} \%$ of MBA, the EE \% were $46.5,50.555 .2$ and $62.3 \%$, respectively. For hydrogels crosslinked with 1,2 , and $3 \mathrm{wt} \%$ of MBA, the $\mathrm{EE} \%$ are $55.2,46.7$, and $41.1 \%$, respectively. Such a decreasing trend in encapsulation efficiency is resulted from reducing the free volume spaces within the polymer matrix associated with an increase in crosslinking density and the ridgidty of the copolymeric hydrogels.

\section{In vitro cumulative drug release studies}

Drug release kinetics was analyzed by plotting the cumulative release values, $\left(M_{t} / M_{\infty}\right)$ versus time, where, $M_{t} / M_{\infty}$ is a fraction of drug released at time ' $t$ '.

The poly(NIPA-co-APA) copolymeric hydrogels were used to study the release behavior of $\mathrm{KT}$ in $\mathrm{pH} 1.2$ and 7.4.

\section{Effect of $N$-acryloyl-L-phenylalanine (APA) content}

Effect of APA content in the hydrogel polymers was studied at constant loading of $1 \mathrm{wt} \% \mathrm{KT}$. The hydrogels prepared with different amounts of APA showed release trends up to $>80 \%$ of cumulative release within $\sim 10 \mathrm{~h}$ (Fig. $5(a))$. A systematic increase in the $\%$ cumulative release was observed with increasing the composition of APA. The three-dimensional networks in the hydrogel polymers swelled systematically more with increasing amount of APA, probably due to the ionization of crosslinked chains. Therefore, the relaxation responses of the polymer chains made an increase of dimension of the polymer coil, and further a significant increase in molecular volume of the overall hydrated polymer matrix. Noteworthy, the nature of release profiles remains almost identical for all the matrices containing different amount of APA indicating that swelling of APA has a linear relationship with their release profiles. 

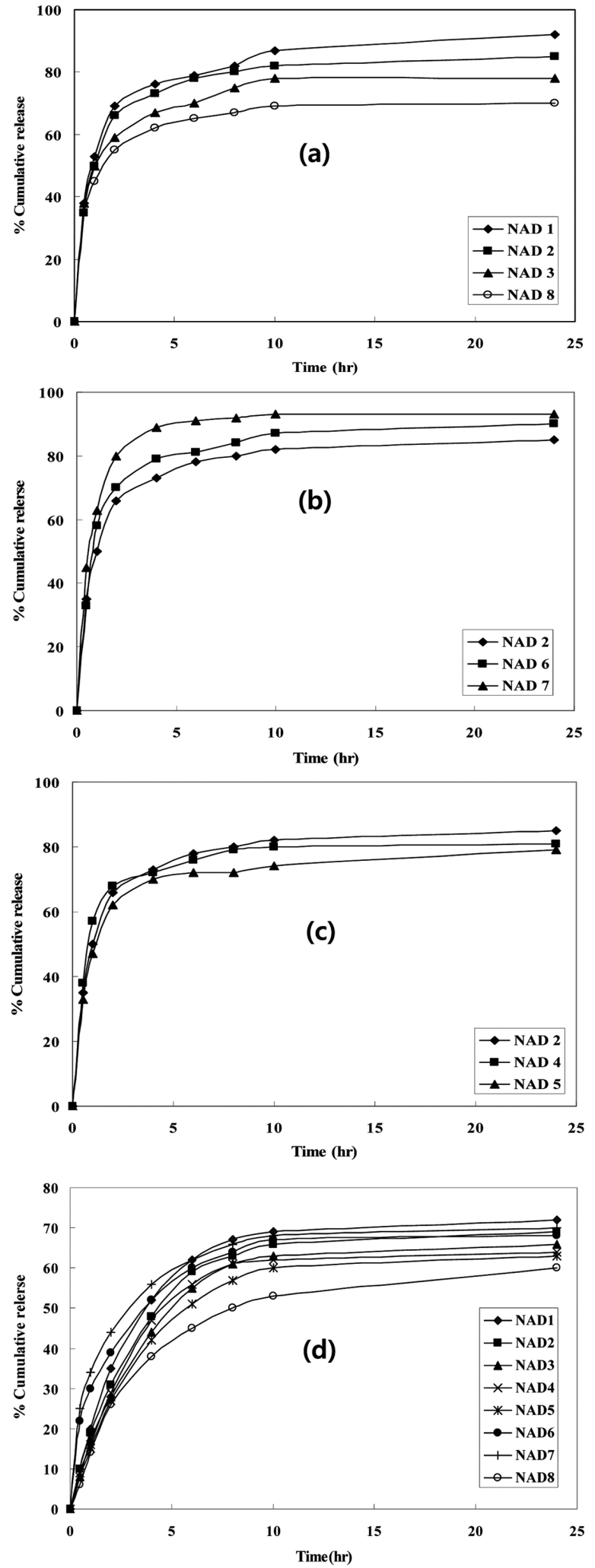

Figure 5. Percentage of cumulative drug release of poly(NIPAco-APA) hydrogels containing (a) different amount of APA, (b) different amount of KT, and (c) different amount of crosslinking agent in $\mathrm{pH} 7.4$ and (d) in $\mathrm{pH} 1.2$ at $37^{\circ} \mathrm{C}$.

\section{Effect of drug loading}

Fig. 5(b) displays the drug release profiles of drug-loaded hydrogels with different amounts of drug loadings (50, 100 , and $150 \mathrm{mg}$ ). The effect of drug loading on the release rates was investigated for three formulations, NAD 2, 6 , and 7 (Table 1).

It was observed that the drug release rates were slower for the formulations containing lower amount of the drug and vice versa.

The drug in the hydrogels might act as inert filler by occupying the free volume of the swollen hydrogel. This could have created a twisted path for water molecules to permeate, however, the degree of twisting depends upon the volume fraction of the filler. ${ }^{27}$

\section{Effect of crosslinking agent}

Typical plots between \% cumulative release data versus time are illustrated in Fig. 5(c) by varying the amounts of MBA (i.e., 1, 2, and $3 \mathrm{wt} \%$ ) at constant drug loading. The $\%$ cumulative release was relatively large at the lower amount of MBA ( $1 \mathrm{wt} \%$ ), compared with those at higher amount of MBA (3 $\mathrm{wt} \%$ ), probably due to the polymeric chains become rigid at higher concentration of MBA owing to the contraction of microvoids.

\section{Effect of pH}

The cumulative drug release experiments of poly(NIPA-coAPA) hydrogels were performed in $\mathrm{pH} 1.2$ and 7.4 dissolution media at $37^{\circ} \mathrm{C}$. Fig. 5 indicates a considerable increase in the cumulative drug release for all the hydrogels when $\mathrm{pH}$ was increased from 1.2 to 7.4. It can be seen that the poly(NIPA-co-APA) hydrogels have shown extensive drug release rates than the pure NIPA hydrogels. However, it should be remarked that there is a drastic difference in the drug release rates of the formulated blend hydrogels in $\mathrm{pH}$ 1.2 and 7.4, implying the drug release of the poly(NIPAco-APA) hydrogels depends upon the nature of the polymer matrix as well as $\mathrm{pH}$ of the media. For instance, in $24 \mathrm{~h}$, only $70 \%$ of drug was released in $\mathrm{pH} 1.2$ (Fig. 5(d)) and $90 \%$ was released in $\mathrm{pH} 7.4$. In the case of the hydrolyzed blend polymer, it is likely that there is a complexation of carboxylic acid groups of APA, but there could be a deformation of the complex (NH-COO-) formed in $\mathrm{pH}$ 1.2.

\section{CONCLUSION}

Copolymeric hydrogels, poly(NIPA-co-APA), based on NIPA and APA were prepared by redox polymerization method and characterized by differential scanning calo- 
rimetry, X-ray diffractometry, and scanning electron microscopy. All hydrogels swelled slowly in $\mathrm{pH} 1.2$ and 7.4 at $37^{\circ} \mathrm{C}$ and reached the equilibria within $24 \mathrm{~h}$. The percentage of swelling ratio of the hydrogels was increased from 44 to 1652 with increasing the amount of APA (from 0 to $300 \mathrm{mg}$ ) and the KT contents. Effect of $\mathrm{pH}$ on swelling kinetic studies revealed that percentage of swelling ratio decreases by decreasing the $\mathrm{pH}$ due to the formation of intermolecular hydrogen bonds. The drug KT was dispersed at the molecular level in the polymer matrix and no crystals were found in the drug-loaded matrices. The amounts of matrix crosslinking agent, drug-loading, and APA content of the matrix influenced the release of KT. The drug release profile was varied (up to $10 \mathrm{~h}$ ) depending upon the nature of the matrix in gastro intestinal tract (GIT) disease conditions.

Acknowledgments. This work was supported by the research project of Changwon National University (20122014).

\section{REFERENCES}

1. Raman, K.; Ramamoorthy, R.; Reddy, B. S. R. React. Funct. Polym. 2005, 62, 215.

2. Vaidya, A. A.; Lele, B. S.; Kulkarni, M. G.; Mashelkar, R. A. Biotechnol. Bioeng. 1999, 64, 418

3. Deshmukh, M. V.; Vaidya, A. A.; Kulkarni, M. G.; Rajamohanan, P. R.; Ganapathy, S. Polymer 2000, 41, 7951.

4. Puskas, J. E.; Chen, Y.; Dahman, Y.; Padavan, D. J. Polym. Sci. Part A: Polym. Chem. 2004, 42, 3091.

5. Vyavahare, N.; Kohn, J. J. Polym. Sci. Part A: Polym. Chem. 1994, 32, 1271.

6. Nathan, A.; Zalipsky, S.; Kohn, J. J. Bioact. Compat. Polym. 1994, 9, 239.

7. Lin, H. B.; Sun, W.; Mosher, D. F.; García-Echeverría, C.; Schaufelberger, K.; Lelkes, P. I.; Cooper, S. L. J. Biomed. Mater. Res. 1994, 28, 329.

8. Salhi, F.; Lam, J. W. Y.; Cheuk, K. K. L.; Cha, J. A. K.; Tan, B. Z. Polymer Preprint 2000, 41, 1185.

9. Song, H.; Chu, C. C. J. Appl. Poly. Sci. 2012, 124, 3840.

10. Sato, M.; Mutsumi Inata, M.; Yamaguchi, I. J. Appl. Poly.
Sci. 2012, 126, E298.

11. Angiolini, L.; Caretti, D.; Giorgini, L.; Salatelli, E.; Altomare, A.; Carlini, C.; Solaro, R. Polymer 1998, 39, 6621.

12. Ramesh Babu, V.; Kim, C.; Kim, S.; Ahn, C. Lee, Y. I. Carbohyd. Polym. 2010, 81, 196.

13. Erbil, C.; Aras, S.; Uyanik, N. J. Polym. Sci. Part A: Polym. Chem. 1999, 37, 1847.

14. Vesterinen, E.; Dobrodumov, A.; Tenhu, H. Macromolecules 1997, 30, 1311.

15. Chen, G.; Hoffman, A. S. Nature 1995, 373, 49.

16. Serizawa, T.; Uemura, M.; Kaneko, T.; Akashi, M. J. Polym. Sci. Part A: Polym. Chem. 2002, 40, 3542.

17. Wang, M.; Fang, Y.; Hu, D. React. Funct. Polym. 2001, 48, 215.

18. Yan, L.; Zhu, Q.; Kenkare, P. U. J. Appl. Poly. Sci. 2000, 78, 1971.

19. Krishna Rao, K. S. V.; Vijaya Kumar, N. B.; Subha, M. C. S.; Sairam, M.; Aminabhavi, T. M. Carbohyd. Polym. 2006, 66, 333.

20. Cui, Z. F.; Guan, Y. X.; Chen, J. L.; Yao, S. J. J. Appl. Poly. Sci. 2005, 96, 1734.

21. Grøttum, J. A.; Erikson, U.; Grasdalen, H.; Staurnes, M. Comparative Biochemistry and Physiology Part A: Molecular \& Integrative Physiology 1998, 120, 469.

22. Shin, B. C.; Jhon, M. S.; Lee, H. B.; Yuk, S. H. Eur. Polym. J. 1998, 34,171.

23. Soppimath, K. S.; Aminabhavi, T. M.; Kulkarni, A. R.; Rudzinski, W. E. J. Controlled Release 2001, 70, 1.

24. Casolaro, M.; Paccagnini, E.; Mendichi R.; Ito, Y. Macromolecules 2005, 38, 2460.

25. Baljit, S. Int. J. Pharm. 2007, 334, 1.

26. Peppas, N. A. Bioavailability and the Pharmacokinetic Control of Drug Response; Wiley: New York, 1980.

27. Pharmacopoeia of India; Controller of publications: Delhi, India, 1985.

28. Dogu, Y.; Okay, O. J. Appl. Poly. Sci. 2006, 99, 37.

29. Gundogan, N.; Melekaslan, D.; Okay, O. J. Appl. Poly. Sci. 2012, 94, 135.

30. Peppas, N. A.; Mikos, A. G. Preparation Methods and Structure of Hydrogels. In Hydrogels in Medicine and Pharmacy; Peppas, N. A., Ed.; CRC Press: Boca Raton, Florida, 1986; pp $1-25$.

31. Allan, S. H. Adv. Drug Delivery Rev. 2002, 54, 3.

32. Peppas, N. A.; Bures, P.; Leobandung, W.; Ichikawa, H.; Eur. J. Pharm. Biopharm. 2000, 50, 27. 